\title{
Noninvasive mechanical ventilation and bron- choscopy: advantages and limits of a synergistic strategy
}

Raffaele Scala

Pulmonology and Respiratory Intensive Care Unit, "S. Donato" Hospital, Arezzo, Italy

Address for correspondence:

Raffaele Scala, MD, FCCP

Pulmonology and Respiratory Intensive Care Unit, "S. Donato" Hospital, Via Nenni 8, 52100 Arezzo, Italy E-mail: raffaele_scala@hotmail.com

\section{Summary}

Bronchoscopy and noninvasive mechanical ventilation (NIMV) are largely applied in respiratory and general intensive care units. Bronchoscopy plays a crucial role for the diagnosis of lung infiltrates of unknown origin and the treatment of bronchial mucous plugging, massive haemoptysis and airways obstruction of different etiologies in critical patients. NIMV is the first-choice ventilatory strategy for acute respiratory failure (ARF) of different causes as it could be used as prevention or as alternative to conventional mechanical ventilation (CMV) via endotracheal intubation (ETI). Some scenarios represent contraindications for these techniques, like severe hypoxemia for bronchoscopy and accumulated tracheo-bronchial secretions for NIMV. In these contexts, ETI is not risk-free. In this Chapter, the rationale, the clinical indications, the scientific evidence, the practical issues and the risks for the synergistic use of bronchoscopy during NIMV are reported.

KEY WORDS: noninvasive ventilation, bronchoscopy, acute respiratory failure, endotracheal intubation, pneumonia, respiratory intensive care unit.

\section{Introduction}

Among the procedures that are routinely used in both general (ICU) and respiratory intensive care unit (RICU), bronchoscopy and noninvasive mechanical ventilation (NIMV) constitutes a "must" for the clinicians who have to face challenging scenarios in critically ill patients. Bronchoscopy is a fundamental technique for the management of respiratory diseases in the acute setting $(1,2)$. In fact, it's not a mere coinci- dence that the first application of bronchoscopy, which dates back to the end of nineteen century, was due for an emergent removal of an inhaled foreign body in a subject with a life-threatening respiratory distress (3, 4 ). Bronchoscopy provides the visualization of the upper airway and initial divisions of the tracheobronchial tree and allows for samples to be taken from the trachea, bronchi, mediastinum and lung parenchyma. Bronchoscopy could be performed both with flexible and rigid instruments. Flexible bronchoscopy (FBO) is widely used both in and out the ICU/RICU setting thanks to its numerous advantages: less invasivity, larger exploration of bronchial tree, shorter learning curve in the training $(1,2,5)$. As a matter of fact, FBO represents a well-defined step in the diagnostic flowchart of severe community-acquired (6) and hospitalacquired pneumonia (7), as well as acute interstitial lung diseases (8). This is also due to the wide range of its ancillary diagnostic procedures, such as bronchoalveolar lavage (BAL), protected specimen brush (PSB), trans-bronchial need aspiration (TBNA), transbronchial lung biopsy (TBLB) and endobronchial ultrasound (1). Even if of less large application rigid bronchoscopy (RB) keeps a crucial role in performing interventional procedures (laser, argo-plasma, stent position) in critical patients with tracheal and/or main bronchial stenosis, massive haemoptysis and inhaled foreign body especially in pediatric patients $(3,4)$. NIMV is the oldest technique reported in the literature to deliver artificial ventilation without the need of an artificial airway (i.e. endotracheal tube or tracheostomy cannula). NIMV includes negative and positive pressure ventilatory techniques which have been shown to achieve the "goals of mechanical ventilation" (i.e. unload respiratory muscles, improve pulmonary gas exchange, increase alveolar ventilation) without the risk of CMV-correlated complications (9).

Negative pressure NIMV (npNIMV) such as the ventilation via "iron lung", supports ventilation by exposing the surface of the chest wall to subatmospheric pressure during inspiration; whereas, expiration occurs when the pressure around the chest wall increases and either becomes atmospheric or remained at negative values lower than those set in inspiration (10). In the opposite, positive pressure NIMV (ppNIMV), which is administered by means of noninvasive interfaces (i.e. oronasal and total face mask, nasal mask and pillows, helmet, mouthpiece), supports the patient's breathing by increasing the inspiratory pressure over the atmospheric level then allowing passive exhalation, either to atmospheric pressure or to a pre-set end-expiratory positive pressure (PEEP) (11).

Because of the tremendous technologic improvement 
of ventilators (12) and interfaces (13), positive-pressure ppNIMV has became the first-line ventilatory treatment in different patterns of ARF (i.e. acidotic chronic obstructive pulmonary disease [COPD] exacerbations, cardiogenic pulmonary edema, immunosoppressed conditions) because, compared to standard medical therapy, it reduces the rate of ETI and its complication, as well as the mortality and length of stay in hospital (9). Conversely, nowadays npNIMV, mainly delivered by "iron lung", is performed only by few expert centers to manage selected patients (i.e. COPD exacerbations non-respondent to ppNIMV, respiratory distress syndrome and weaning difficulty especially in paediatric patients) (10).

In this Chapter, the rationale, the clinical indications, the scientific evidence, the practical issues and the risks for the synergistic use of bronchoscopy during NIMV are reported.

Pitfalls of bronchoscopy and noninvasive ventilation

Despite its large application in ICU/RICU, bronchoscopy can be challenging and risky in non-intubated patients with ARF. This is the case of diagnostic FBO in subjects admitted in ICU/RICU for severe hypoxemia due to lung infiltrates of unknown origin. Performing bronchoscopy in critically ill patients presents potential complications that can be related with proce- dure itself, patient-related factors and of bronchoscopist's experience. In this context, the crucial point is the cardio-pulmonary patho-physiologic impact of FBO that has been well investigated since long time ago $(14,15)$ (Figure 1).

First of all, the bronchoscope occupies $10-15 \%$ of the normal tracheal lumen, causing an increase in resistance of the airway and a drop in tidal volume with the augmentation of work of breathing. Consequently, the patient develop a rapid-shallow breathing pattern with a risk of impending respiratory muscle fatigue, worsening of gas exchange and need of mechanical ventilation. Due to the incomplete expiratory lung emptying, FBO could facilitate "air trapping" with a generation of intrinsic PEEP, which is particularly deleterious in $\operatorname{COPD}(1,2,4,14,15)$.

Hypoxemia occurs with insertion of the bronchoscope into the trachea and becomes worse when BAL is performed as consequence of ventilation-perfusion (V/Q) mismatch $(14,15)$. The application of suction through the bronchoscope channel lowers airway pressure at the end of expiration, facilitating early alveolar closure and hypoxemia. As a matter of fact, FOB induces arterial oxygen pressure $(\mathrm{PaO} 2)$ to decrease between 10 and $20 \mathrm{mmHg}(1,2,15,16)$. The use of analgo-sedation during FBO to reduce patient's discomfort may worsen gas exchange furthermore through drug-induced hypoventilation (17). These pulmonary changes persist after the procedure is completed, and the time

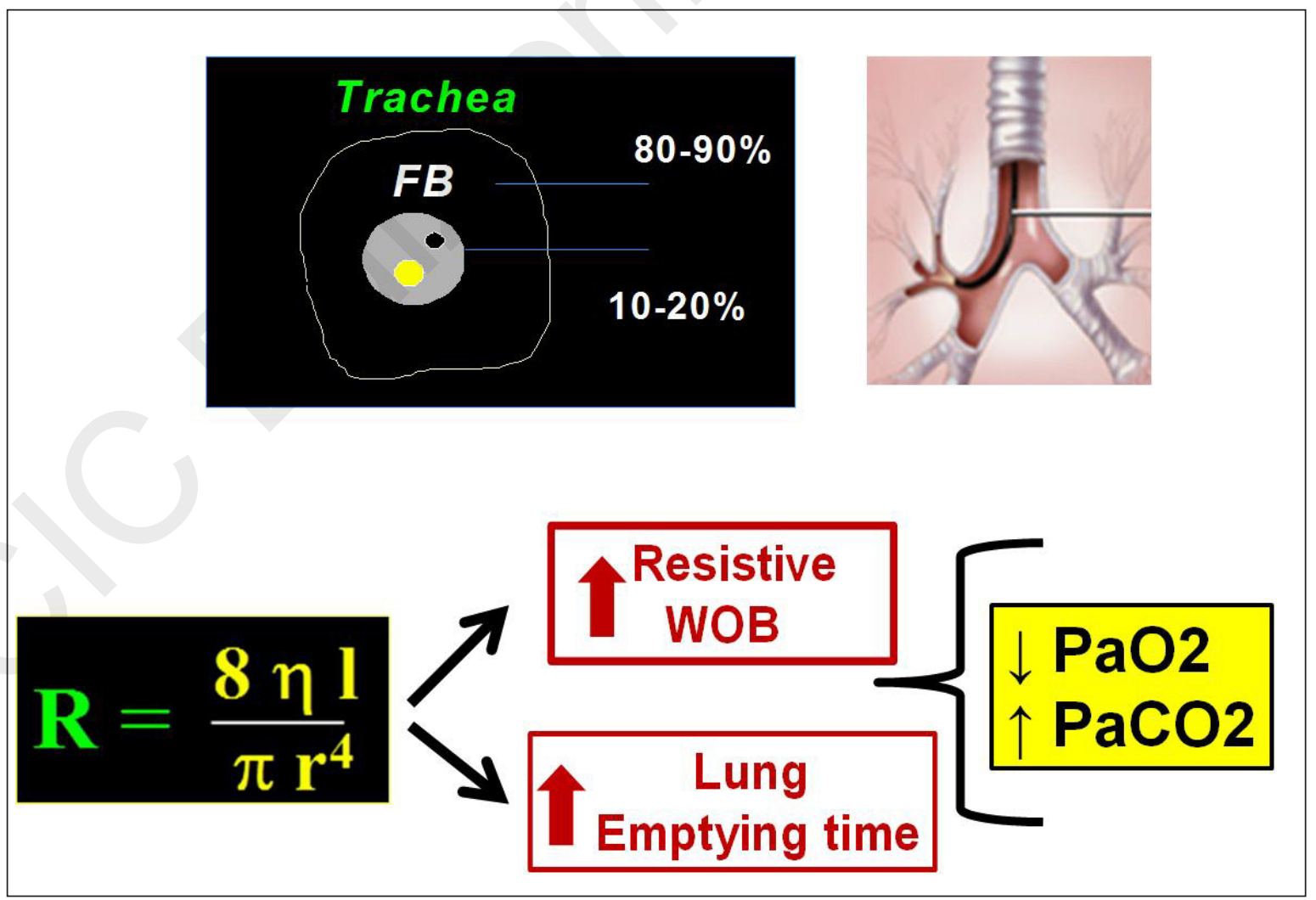

Figure 1 - Patho-physiologic effects of bronchoscopy on respiratory system. 
Table 1 - Advantages and disadvantages of bronchoscopy and noninvasive ventilation.

\begin{tabular}{|c|c|c|}
\hline & BRONCHOSCOPY & NONINVASIVE VENTILATION \\
\hline \multicolumn{3}{|l|}{ Advantages } \\
\hline & -Diagnosis in suspected pneumonia & -Improving gas exchange \\
\hline & -Diagnosis in diffuse lung diseases & -Reduced work of breathing \\
\hline & -Clearing of mucous from airway & -Reduced heart work-load \\
\hline & -Treatment of airways obstruction & -Avoidance of ETI and CMV \\
\hline \multicolumn{3}{|l|}{ Disadvantages } \\
\hline & -Increased airway's resistance & -Failure in case of burden of secretion \\
\hline & -Increased work of breathing & \\
\hline & -Worsening of gas exchange & \\
\hline & -Increased heart work-load & 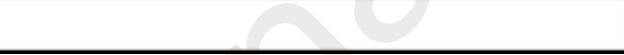 \\
\hline
\end{tabular}

that the gas exchange takes to normalize ranges from 15 min in normal subjects to several hours in patients with lung disease $(14,15)$. All this justifies the use of supplemental $\mathrm{O} 2$ in patients at risk for oxygen desaturation undergoing FBO (18). Furthermore, bleeding and pneumothorax complicating bioptic procedures may precipitate suddenly life-threatening deterioration of blood gases $(1,2)$.

FOB-induced sympathetic stimulation and hypoxemia could lead to an increase in heart rate and cardiac output with augmented heart's oxygen consumption. Decreased intra-thoracic pressure caused by the augmented respiratory muscle efforts (i.e. deeper negative trans-diaphragmatic swings during inspiration) produce an increase in both right ventricular pre-load and left ventricular after-load. Consequently, FBO may trigger cardiac arrhythmias in $11-40 \%$ of the cases, and, less frequently, cardiogenic pulmonary edema and acute coronary syndrome, especially in patients with pre-existent heart diseases $(19,20)$.

The findings of the clinical studies which have evaluated the need of ETI as a consequence of cardio-pulmonary deterioration in severely hypoxemic patients undergoing diagnostic FBO are contrasting (16). It was reported that BAL performed in ICU did not significantly increase ETI requirements in critically ill cancer patients with ARF, compared with noninvasive diagnostic tests used for identifying the cause of ARF (21). In a large French prospective study evaluating the safety of FBO on 169 non-intubated hypoxemic patients (PaO2/FiO2 $<300 \mathrm{mmHg}$ ), 1/3 of the bronchoscopic procedures were complicated by an increase in ventilatory support: NIMV for oxygen-supported patients and CMV for non-invasively ventilated patients. ETI was required in $15 \%$ of the procedures overall but this complication occurred 2 hours after FBO in only $4 \%$ of the cases (22). However, American Thoracic Society recommended avoiding $\mathrm{FBO}$ and $\mathrm{BAL}$ in patients with hypoxemia that cannot be corrected to at least a $\mathrm{PaO} 2$ of $75 \mathrm{~mm} \mathrm{Hg}$ or to an oxygen saturation
(SpO2) of $\geq 90 \%$ with supplemental oxygen $(1,23)$. In these higher risk patients, when noninvasive diagnostic tests are not conclusive, avoiding FBO means being compelled to use empirical treatment. As a consequence, when bronchoscopy is mandatory, only CMV can assure adequate ventilation during the maneuver. Unfortunately, as previously underlined, CMV is not free of risks as it exposes these fragile patients to complications related to ETI, baro- or volutrauma, loss of airway defense mechanisms, and difficulties in weaning after the extubation $(24,25)$.

Cardio-pulmonary patho-physiologic changes observed during FBO are more exaggerated during intervention procedures performed with RB especially because of greater degree of airway obstruction caused by the bronchoscope, prolonged suctioning, low inspiratory fraction of $\mathrm{O} 2$ (FiO2) (to prevent laser combustion), and respiratory depression resulting from deep analgo-sedation $(3,4,26,27)$.

Despite its increasing clinical application in critical respiratory patients, the failure rate of ppNIMV failure varies between 5 and $60 \%$, depending on numerous factors, including the etiology of ARF, excessive secretions, hypercapnic encephalopathy $(\mathrm{HE})$, agitation, patient-ventilator asynchrony, sleep disturbances $(9$, $24,28)$. Unsuccessful ppNIMV was found to be independently associated with death, especially in patients with de novo hypoxemic ARF (29). Among all, weak cough reflex with an excessive burden of secretions is one of the most important cause of immediate ( $>1$ hour) ppNIMV failure (28). This is due to the noninvasive interfaces which do not allow a direct access into the airways (Table 1). A rate of ppNPPV failure of $61 \%$ was reported in 23 ARF patients showing copious secretions (30). Moreover, in two series of COPD exacerbations with moderate-severe $\operatorname{HE}(31,32)$ the inefficient clearance of secretions caused 33 and $43 \%$ of all ppNIMV failures, respectively. Conti et al. (33) showed that in 2 of the 9 COPD patients who required ETI within 2-6 hours of ventilation, ppNIMV failed for 
the difficult management of copious secretions.

\section{Rationale for the combined use of NIMV and FBO}

In presence of the above reported limitations for the use of bronchoscopy (diagnostic and therapeutic procedures in severe ARF) and

Noninvasive ventilation may help performing a safe bronchoscopy in patients with acute respiratory failure; but also FBO may increase the chance of success in patients at risk of NIMV failure. ppNIMV (noninvasive support in case of accumulated secretions and depressed cough) in critical respiratory patients, ETI seems to be the last viable strategic resort. However, clinicians should carefully consider the risk of CMV-correlated complications which is particularly high in fragile patients (i.e. advanced age and multiple comorbidities, immunocompromised status, underlying lung diseases with poor prognosis such as idiopathic pulmonary fibrosis and malignancies) $(1,2,9,24,25$, 34). Ethical issues (e.g. "do-not intubate" and "do-not resuscitate" status) and economic aspects (e.g. limited beds in ICU/RICU) make more challenging the decision of submitting high-risk ARF patients with uncertain prognosis to an "escalating" therapeutic strategy involving invasive procedures (e.g. ETI plus CMV, renal replacement therapy, aggressive hemodynamic support) (35). Are there alternative strategies to ETI for the safe and effective use of bronchoscopy and NIMV when the two techniques raise worrying concerns?

There is a strong patho-physiologic rationale in combining bronchoscopy and NIMV for the management of respiratory critical patients because the limitations of one of the two techniques may be counterbalanced by the properties of the other one. In other words, NIMV may be of "help" to perform a safe bronchoscopy in ARF patients; on the other hand, FBO may increase the chance of success in patients at risk of ppNIMV failure $(25,34)$.

NIMV, both if delivered with positive or negative pressure techniques, is able to prevent and correct the cardiopulmonary alterations induced by bronchoscopy throughout three mechanisms: 1) compensation of the bronchoscope-correlated extra resistive work of breathing by means of the unload of respiratory muscles leading to a more favorable breathing pattern and diaphragmatic load-force relationship; 2) improvement of pulmonary gas exchange due to a better V/Q ratio and the correction of hypoventilation; 3) counterbalance of the increased heart work-load by means of a marked relief of respiratory muscle efforts with a reduced negative inspiratory intra-thoracic pressure. Moreover, keeping the patient on NIMV after bronchoscopy may prevent the risk of derangement of lung function which could last several hours after the procedure (25).

Thanks to the possibility of clearing the airways in the early phases of ppNIMV, FBO may improve ventilation and, therefore, reduce the need for ETI in patients with an unfavourable balance between an excessive burden of secretions and an inefficient spontaneous clearance after the failure of chest physiotherapeutic techniques (25).

Three different acute scenarios of synergistic interaction between FBO and ppNIMV may be depicted in ICU/RICU: 1) patients on O2-therapy who undergo diagnostic FBO under ppNIMV assistance (prevention of a ventilatory support in spontaneously breathing patients); 2) patients already on ppNIMV who undergo diagnostic FBO under ventilation (prevention of CMV in ppNIMV supported patients); 3) patients requiring ETI for an excessive burden of bronchial secretions who undergo early therapeutic and diagnostic FBO during ppNIMV (alternative to mandatory CMV in ppNIMV plus FBO supported patients) or patients requiring CMV through RB who undergo interventional procedures with the support of npNIMV (alternative to mandatory CMV during RB in npNIMV supported patients) (Figure 2).

\section{Clinical evidence of NIMV-bronchoscopy synergy}

The majority of the published studies has used ppNIMV to prevent respiratory deterioration and ETI in spontaneously breathing hypoxemic patients undergoing FBO who do not still required the criteria for starting ppNIMV $(16,25)$ (Table 2). Antonelli et al. (36) were the first to perform ppNIMVassisted FBO with BAL in 8 immunocompromised severely hypoxemic patients $(\mathrm{PaO} 2 / \mathrm{FiO} 2 \leq 100 \mathrm{~mm} \mathrm{Hg})$ admitted in ICU for suspected pneumonia. The use of ppNIMV was associated with significant improvements in $\mathrm{PaO} 2 / \mathrm{FiO} 2$ and $\mathrm{SpO} 2$ during bronchoscopy. FBO with NPPV was well tolerated, and no patient required ETI. Two patients died 5 to 7 days after FBO from unrelated complications of the underlying illness. A causative pathogen was identified by BAL in all patients and 6 of them responded to treatment and survived hospital admission. In a subsequent study, Da Conceiçao et al. (37) investigated the feasibility of ppNIMV-assisted FBO in a 10 COPD patients with hypoxemic-hypercapnic ARF $(\mathrm{PaO} 2=53+/-13 \mathrm{mmHg}$; $\mathrm{PaCO} 2=67+/-11 \mathrm{mmHg}$ ) admitted to ICU for pneumonia. SpO2 increased from $91 \pm 4.7 \%$ at baseline to $97 \pm 1.7 \%$ during FBO under ppNIMV, while blood gases did not change during the hour following FBO. The procedure was performed without complications and was well tolerated in 8 patients. No patient required ETI within 24 hours and all patients survived. In the first randomized controlled trial (RCT) conducted on 30 patients with $\mathrm{PaO} 2 \leq 125 \mathrm{mmHg}$ under high-flow O2-mask (i.e. $10 \mathrm{l} / \mathrm{min}$ ) requiring diagnostic $\mathrm{FBO}$ for suspected pneumonia, Maitre et al. (38) showed significantly higher $\mathrm{SpO} 2$ values during $\mathrm{FBO}$ and 30 min- 


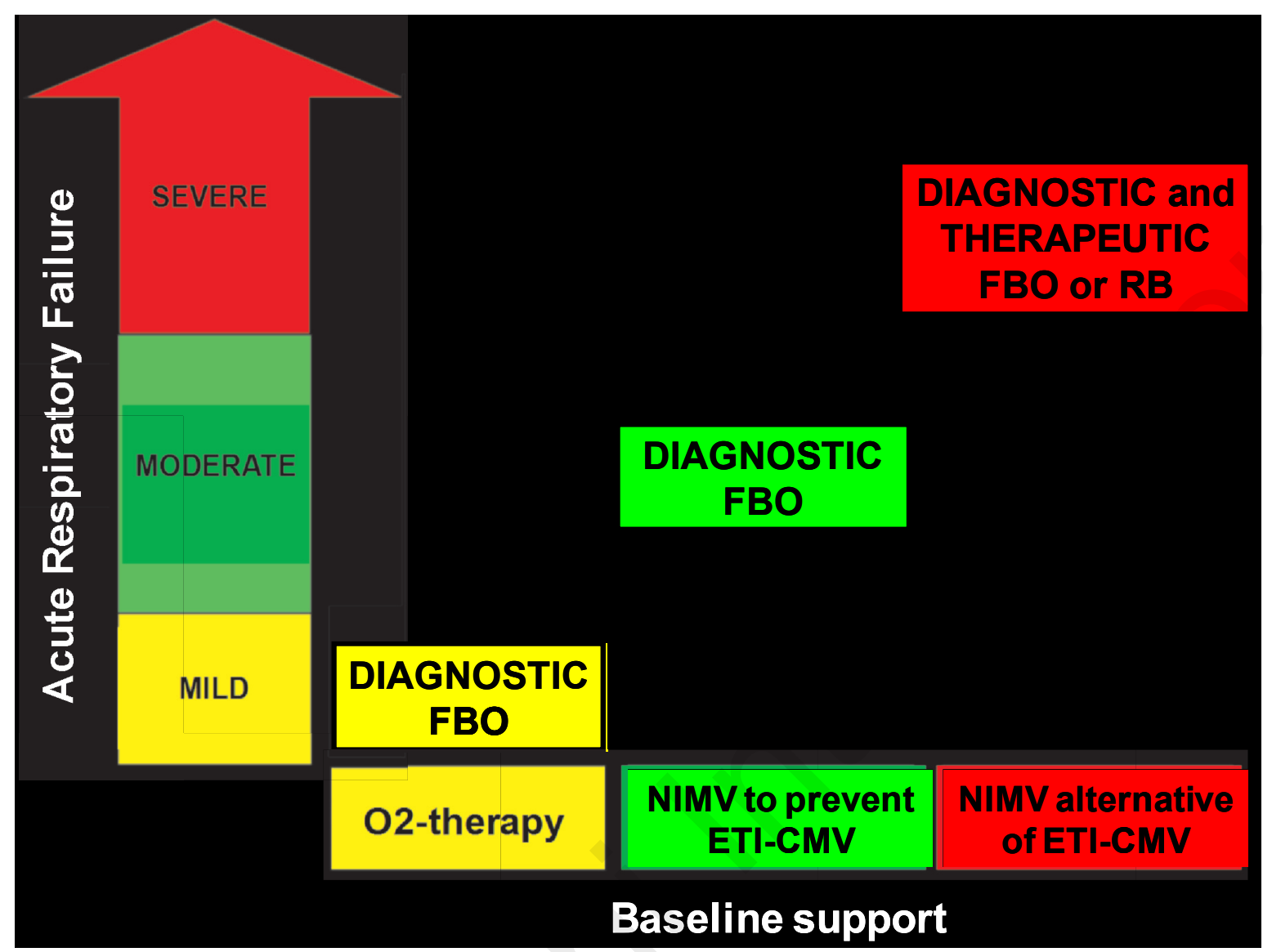

Figure 2 - Potential scenarios for synergistic application of bronchoscopy and noninvasive ventilation according to the severity of acute respiratory failure, the baseline support of the patients and the purposes of bronchoscopy.

Table 2 - Studies on the combined use of FBO and ppNIMV in acute respiratory failure.

\begin{tabular}{|c|c|c|c|c|c|c|c|}
\hline Author, year & Study & n.pts & ARF Pattern & Support pre-FBO & Indication FBO & FBO procedure & ETI $(\%)$ \\
\hline Antonelli, 1996 & Prospective & NMV: 8 & Hypoxemic & Oxygen & Diagnostic & $\mathrm{BAL}$ & NIMV: 0 \\
\hline Da Conceicao, 2000 & Prospective & NMV: 10 & Hypox-Hypercap & Oxygen & Diagnostic & $\mathrm{BAL}$ & NIMV: 0 \\
\hline Maitre, 2000 & RCT & $\begin{array}{l}\text { CPAP: } 15 \\
\text { O2: } 15\end{array}$ & Hypoxemic & Oxygen & Diagnostic & BAL, br. biopsy & $\begin{array}{l}\text { CPAP: } 6,7 \\
\text { O2: } 46,7\end{array}$ \\
\hline Antonelli, 2002 & RCT & $\begin{array}{l}\text { NMV: } 13 \\
\text { O2: } 13\end{array}$ & Hypoxemic & Oxygen & Diagnostic & BAL & $\begin{array}{l}\text { NIMV: } 7,7 \\
\text { O2: } 15,4\end{array}$ \\
\hline Criner, 2010 & Prospective & NMV: 35 & Hypoxemic & Oxygen & $\begin{array}{l}\text { Diagnostic } \\
\text { Therapeutic }\end{array}$ & $\begin{array}{l}\text { BW, PSB,BAL, } \\
\text { br. biopsy }\end{array}$ & 0 \\
\hline Heunks, 2010 & Prospective & NMV: 12 & Hypoxemic & Oxygen & Diagnostic & $\mathrm{BAL}$ & NIMV: 8,3 \\
\hline Scala, 2010 & Case-control & $\begin{array}{l}\text { NMV: } 15 \\
\text { CMV: } 15\end{array}$ & Hypox-Hypercap & NIMV & $\begin{array}{l}\text { Diagnostic } \\
\text { Therapeutic }\end{array}$ & $\mathrm{BAL}$ & NIMV: 20 \\
\hline Baumann, 2011 & Prospective & NMV: 40 & Hypoxemic & NIMV & Diagnostic & BAL & NIMV 10 \\
\hline Clouzeau, 2011 & Prospective & NMV: 23 & Hypoxemic & NIMV & Diagnostic & $\mathrm{BAL}$ & NIMV: 17,4 \\
\hline Agarwall, 2012 & Prospective & NMV: 6 & Hypoxemic & Oxygen & Diagnostic & BAL, TBLB & NIMV: 1,7 \\
\hline
\end{tabular}

utes thereafter with CPAP, compared to oxygen-therapy, using a new device open to the atmosphere (i.e. CPAP-Boussignac). Not only did the patients in the oxygen-group develop hypoxemia during FBO, but also 5 patients in the oxygen-group (compared to none in the CPAP-group) required ventilatory assistance (1 ppNIMV and $4 \mathrm{CMV}$ ) within 6 hours following the procedure $(p=0.003)$. Etiology of lung infiltrates was determined in 18 patients: pneumonia with identification of the infectious agent (n.14), carcinomatous lym- 
phangitis (n.2; one by BAL and one by bronchial biopsy), fat embolism and drug-induced hypersensitivity pneumonia (n.2). Subsequently, in another RCT involving 26 patients with nosocomial pneumonia and $\mathrm{PaO} 2 / \mathrm{FiO} 2 \leq 200 \mathrm{mmHg}$, Antonelli et al. (39) evaluated the effectiveness and the safety of ppNIMV versus conventional oxygen-therapy before, during and after FBO. Application of ppNIMV was associated with increase in $\mathrm{PaO} 2 / \mathrm{FiO} 2$ by $82 \%$, in contrast to decline in $\mathrm{PaO} 2 / \mathrm{FiO} 2$ by $10 \%$ in the oxygen-group during $\mathrm{FBO}$. Furthermore, in the ppNIMV group the $\mathrm{PaO} 2 / \mathrm{FiO} 2$ remained higher, the heart rate was lower, and no reduction in mean arterial pressure in comparison to a $15 \%$ decrease from the baseline in the oxygen-group 60 min after FBO. Causative microbiologic pathogen was identified with BAL in 18 cases. One patient in the ppNIMV group and two patients in the oxygen-group required non-emergent ETI. Four patients in the ppNIMV group and seven patients in the oxygengroup died from complications of their underlying disease 5 to 7 days after study entry. The same group (40) also found the helmet for delivering NIMV to be safe in avoiding gas exchange deterioration in 4 hypoxemic patients. Chiner et al. (41) evaluated nasal mask for delivering ppNIMV while the FBO was performed orally using a bite block sealed with an elastic glove finger in 35 patients with a mean $\mathrm{PaO} 2 / \mathrm{FiO} 2$ of $168 \mathrm{mmHg}$. A total of 35 bronchoaspirates, $21 \mathrm{PSB}, 11$ $\mathrm{BAL}$ and 8 bronchial biopsies were done. In contrast to other studies, patients developed hypoxemia during the procedure (mean SpO2 86\%) probably for excessive mouth-air leaks during nasal-NIMV. The clinical course was favorable in $66 \%$; there was a relatively high rate of CMV (11\% of the cases occurring $5 \pm 4$ days after FBO) and in-hospital mortality (33\% of the cases occurring $3 \pm 2$ days after FBO), mainly correlated to the underlying disease. Heunks et al. (42) reported the use of total-face mask for delivering NIMV during diagnostic FBO in 12 hypoxemic patients (mean $\mathrm{PaO} 2 / \mathrm{FiO} 2=192 \pm 23 \mathrm{mmHg}$ ). The procedure was successful in all patients; in only one patient $\mathrm{SpO} 2$ decreased to $86 \%$ during FBO. Microbiological diagnosis was established in 8 patients.

All the reported studies have shown that ppNIMV is able to ensure adequate gas exchange during FBO in spontaneously breathing hypoxemic patients, thus avoiding ETI.

There is only one study who evaluated the use of FBO in patients with hypoxemic ARF requiring ppNIMV before the procedure. Baumann et al. (43) analyzed 40 hypoxemic patients under ppNIMV (mean $\mathrm{PaO} 2$ / $\mathrm{FiO} 2=176 \pm 54 \mathrm{mmHg}$ ) who underwent FOB for suspected pneumonia. FBO was successfully performed without complications. BAL yielded diagnostic information in $68 \%$ of the patients. $\mathrm{PaO} 2 / \mathrm{FiO} 2$ ratio improved from at the end of FBO after 120 minutes. Four patients $(10 \%)$ required ETI during the first 8 hours after the procedure. Further controlled trials are needed to confirm the advantage of NIMV vs CMV to support $\mathrm{FBO}$ in this clinical scenario.

Agarwal et al. (44) recently published a pilot experience of ppNIMV-assisted FBO for performing BAL and
TBLB in 6 severely hypoxemic patients ( $\mathrm{PaO} 2$ ) $\mathrm{FiO} 2<200 \mathrm{mmHg}$ ) with diffuse interstitial lung diseases. There was significant improvement in respiratory rate and $\mathrm{PaO} 2 / \mathrm{FiO} 2$, and significant decline in heart rate after application of ppNIMV. FBO was well tolerated and all subjects maintained SpO2>92\% during the procedure. One subject required ETI due to haemoptysis. There was no evidence of pneumothorax in any subject. A definite diagnosis was obtained in 5 patients ( $\mathrm{n} .2$ malignancy, n.1 lymphoma, n. 1 sarcoidosis, n.1 pneumocystis pneumonia) only with TBLB, which enabled their successful management. Even if ppNIMV-assisted TBLB is feasible in patients with ARF and diffuse pulmonary infiltrates, this approach should be performed only in centers showing wide experience with both ppNIMV and FBO, as well as with the management of TBLB complications (i.e. pulmonary hemorrhage and pneumothorax). More studies are required to define the balance between utility and risks of NIMV-assisted TBLB in ARF patients.

The last scenario of a synergistic use of the two techniques deals with the feasibility and effectiveness of FBO as early therapeutic tool to avoid ppNIMV failure in ARF patients with an excessive burden of secretions (45). In a matched case-control study, Scala et al. (46) compared 15 acutely decompensated COPD patients with copious secretion retention and $\mathrm{HE}$ due to community-acquired pneumonia undergoing early FBO plus BAL during ppNIMV in an expert RICU with 15 controls receiving CMV in the ICU. Two hours of ppNIMV plus FBO significantly improved gas exchange, sensorium and cough efficiency without major complications (cardiovascular events, emergent ETI, pneumothorax). Improvement in $\mathrm{PaCO} 2$ and $\mathrm{pH}$, as well as hospital mortality, and durations of hospitalisation and ventilation were similar in both groups. ppNIMV significantly reduced serious infectious complications compared with CMV, as well as the need for tracheostomy. Even if this ppNIMV strategy may be a successful alternative to CMV in selected COPD patients within expert units, larger RCTs are necessary to confirm this result and, therefore, to test the efficacy of the FBO-ppNIMV protocol applied to an earlier time-course of COPD decompensations when ETI is not mandatory by comparing ppNIMV alone vs ppNIMV with early FBO (46). A recent experience published by the same group reported the successful management of ppNIMV failure, due to an excessive burden of both secretion and $\mathrm{CO} 2$ in an end-stage COPD patient with exacerbation of hypercapnic ARF, by means of a sequential and integrated use of ppNIMV together with $\mathrm{FBO}$, high-frequency chest wall oscillation and veno-venous extra-corporeal CO2-removal (47).

\section{Special Scenarios}

\section{Difficult intubation}

The combined use of FBO and ppNIMV to perform ETI may be useful in peculiar clinical contexts. ppNIMV is 
effective in supporting spontaneously breathing critical patients during FBO-assisted ETI. On the other hand, FBO could be of help to facilitate ETI in patients who have failed ppNIMV $(16,25,48)$.

In a RCT performed on 32 patients with an anticipated difficult ETI in ear-nose-throat surgery, Bourgain et al. (49) demonstrated that as compared to spontaneous breathing, ppNIMV was more effective to improve ventilation efficiency, guaranteeing higher tidal volumes and lower end-tidal PCO2 values, during FBO performed under propofol anesthesia.

$\mathrm{ETI}$ is particularly risky and difficult in severely hypoxemic patients who deteriorate despite a trial of ppNIMV (29). The lack of clear benefit of ppNIMV in these patients is at least in part due to an increased mortality rate during rescue ETI. In fact, these patients are at risk of developing major complications (e.g. lifethreatening arrhythmias, cardiac arrest) when the mask is removed for intubation (50). Even in centers with high experience, 3 out 21 ARF patients developed cardiac arrest during several attempts of performing ETI after ppNIMV failure (51). Maintaining ppNIMV during ETI may prevent alveolar de-recruitment and derangement of gas exchange. This concept was demonstrated by Bailard et al. (52) in a RCT study conducted on 53 hypoxemic patients requiring $\mathrm{ETI}$ in ICU. The Authors showed that $\mathrm{SpO} 2$ values were significantly greater if pre-oxygenation before ETI was performed with ppNIMV than with conventional nonrebreather bag-valve mask. In the first pilot French study, Da Conceiçao et al. (53) assessed the feasibility and safety of a new technique of FBO-assisted tracheal naso-intubation with ppNIMV delivered via an adapted endoscopic facial mask under conscious sedation in 16 patients with hypoxemic-hypercapnic ARF $(\mathrm{PaCO} 2=64+/-26 \mathrm{mmHg}, \mathrm{PaO} 2 / \mathrm{FiO} 2=142+/-70 \mathrm{mmHg}$ before pp-NIMV) after late ppNIMV failure. SpO2 significantly improved during ETI and remained over $90 \%$ over the procedure. FBO-intubation was performed without any failure or complication. In another US pilot study, Barjaktarevic et al. (54) reported the series of 10 nonconsecutive hypoxemic patients who developed failure of ppNIMV via full-face mask showing a $\mathrm{PaO} 2 / \mathrm{FiO} 2$ ratio lower than $100 \mathrm{mmHg}$; the subjects were orally intubated under the guide of FBO keeping ppNIMV delivered via nasal route. Adopting this new technique, ETI was successfully performed without major complications; $1 / 3$ of the patients developed arterial hypotension mainly correlated with analogo-sedation. Only a mild drop in SpO2 (4.7+/-3.1\%) was reported during the procedure.

These preliminary experiences require the confirm by large RCTs comparing conventional vs FBO-guided ETI under ppNIMV in patients with either predicted or proven difficult direct laryngoscopy or with impending ppNIMV failure due severe hypoxemia.

\section{Obstructive sleep apnea syndrome}

Patients with obstructive sleep apnea syndrome (OSAS) are at high risk of developing severe hypoxemia under and after sedation during surgical or endo- scopic procedures (55). This is particularly true in case of FBO performed under sedation which may precipitate airways collapse and severe hypoxemia in OSAS patients. ppNIMV
ppNIMV counteracts negative inspiratory pressures and hypotonicity of the upper OSAS patients. airway muscles in counteracts negative inspiratory pressures and hypotonicity of the upper airway muscles in these patients $(16,25,56)$. Furthermore, the positive pressure allows for the laryngeal structures to be identified as the device passes the hypopharynx and is introduced through the vocal chords. This aspect is fundamental in OSAS patients with predicted difficult intubation (57).

\section{Pediatric patients}

Pediatric patients are at a high risk for hypoxemia and hypercapnia during FBO due to the smaller diameter and the greater tendency for collapse of their airways (58). Any obstruction due to edema and lesions occupying space will exponentially increase the resistance to gas flow, limiting ventilation by significant reductions in tidal volume, peak inspiratory pressure and expiratory flow (59). In children, CPAP increases the width of the laryngeal space and reduces the tendency toward collapse of the lateral walls of the pharynx and counterbalances
In children, CPAP increases the width of the laryngeal space and reduces the tendency toward collapse of the lateral walls of the pharynx and counterbalances the FBO-induced shallow breathing pattern. the FBO-induced shallow breathing pattern (60). These favorable effects of CPAP are particularly useful in infants with tracheobronchomalacia who are more vulnerable to airways collapse (61).

\section{Interventional procedures during $R B$}

The application of intermittent positive pressure and jet-ventilation are the two standard CMV modes that guarantee effective ventilation during RB. Patients could also be managed with assisted spontaneous breathing. Unfortunately, these ventilatory strategies have important limitations during $\mathrm{RB}$, such as requirement of higher FiO2, ineffective control of ventilatory out-put with risk of acidosis, need for higher doses of opioids, prolonged recovery time $(3,25-27)$.

In two RCTs Natalini et al. $(62,63)$ demonstrated that as compared to both assisted spontaneous breathing and external high-frequency oscillation, npNIMV delivered by a poncho-wrap to support interventional RB procedures under general anesthesia, was associated with less incidence and severity of respiratory acidosis, less requirement of increased $\mathrm{O} 2$ supply, lower use of opioids, shorter recovery time, and less need for assisted-manual ventilation. The applicability of this technique is limited since these favorable results 
were obtained by only one expert Italian center.

\section{Practical issues}

First of all, it should be highlighted that FBO-NIMV procedures must be performed in an ICU/RICU setting, or a standard bronchoscopy room capable of dealing with any cardio-pulmonary complications and with the management of airways $(16,25)$.

Concerning the timing, ppNIMV should be initiated at least 15-20 min before bronchoscopy in the spontaneously breathing patients.

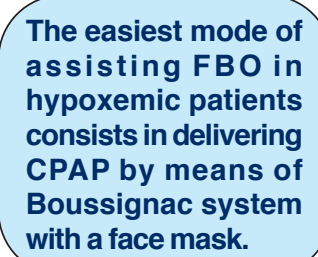

There are no published papers comparing the different ventilatory modes. The easiest mode of assisting FBO in hypoxemic patients consists in delivering CPAP by means of Boussignac system with a face mask

(38). This system accelerated the air molecules in form of microjets that generates a "virtual valve" through a turbulence effect. The gas velocity transforms into pressure depending on the flow of gas provided. Usually, CPAP is initially set at 10 $\mathrm{cmH} 2 \mathrm{O}$ with $\mathrm{FiO} 2$ of 1.0 and then the pressure is eventually increased depending on $\mathrm{SpO} 2$ values. As this CPAP device remains open to the atmosphere, it has the advantage to allow easy maintenance of a positive pressure and CPAP delivery while FBO is being performed (38).

Pressure-support ventilation has been the most commonly used mode of ppNIMV $(9,11,24,25)$. An initial pressure support of $10 \mathrm{cmH} 2 \mathrm{O}$ is recommended during FBO with low PEEP levels (i.e. $5 \mathrm{cmH} 2 \mathrm{O}$ ) (25). With advanced ventilators provided with a double-tube circuit and an accurate ventilatory monitoring, pressure support could be finely titrated in order to achieve an expiratory tidal volume of $8-10 \mathrm{ml} / \mathrm{kg}$ and respiratory rate below $25 \mathrm{~min}-1$. $\mathrm{FiO} 2$ is initially set at 0.5 and then changed to achieve SpO2 values above 90\%; accordingly, an ICU ventilator capable of delivery $\mathrm{FiO} 2$ from high-pressure sources is recommended (12). ppNIMV setting adjustments could be done during the procedure (25):

a) if there is hypoxemia despite $\mathrm{FiO} 21.0$, PEEP may be increased by step of $2 \mathrm{cmH} 2 \mathrm{O}$ until $\mathrm{SpO} 2 \geq 90 \%$ is reached; patients who remain hypoxemic despite high FiO2 and PEPP levels are not good candidate for NIMV-assisted FBO;

b) hypercapnia and respiratory acidosis have to be corrected by raising pressure support levels in order to improve effective alveolar ventilation before FBO;

c) in case of low expiratory tidal volume values, an accurate evaluation of leaks is advised; then, if the mask fitting is fine, pressure support values could be incremented.

Regarding the interfaces, almost all types of masks have been applied for NIMV-assisted FBO. The most widely used are oro-face masks $(13,25)$. Endoscopic face masks usually have two orifices: the first for the administration of gas and the second for the introduction of the bronchoscope. FBO with ppNIMV could be performed with helmet (40) or total-face mask (42). Chiner et al. (41) used a hand-made system of a membrane made out of a latex glove coupled with a bite block and a small incision; this maintains the pressure administered by ventilator through a nasal mask, and FBO may be carried out orally.

Bronchoscope could be introduced through both the nasal and oral route depending on the mask used and the operator's experience (25) (Figure 3). With face masks, nasal or oral pathways can be used. The oral route requires a direct introduction of the bronchoscope through a sealed connector inserted between the mask and the circuit; bending during the entrance of the bronchoscope should be avoided and the use of a mouthpiece is strongly recommended in order to prevent serious damages of the instrument. Conversely, the nasal route, which is usually chosen during routine $\mathrm{FBO}$ for the facilitated access trough the vocal cords, may be really difficult during face-mask NIMV, as the bronchoscope has to be considerably manipulated, which not only prolongs the procedure but may also cause trauma to both the nasal mucosa and the instrument itself. To facilitate easy passage, the bronchoscope is initially passed through the facemask, and the tip of bronchoscope is gently passed through the nose till the vocal cords are visible. With the helmet, either nasal or oral entry could be used, with the patient sitting or in supine decubitus (40). Likewise, the use of Boussignac CPAP allows for oral or nasal entry (38).

Delivery of ppNIMV does not necessarily imply greater sedation. For topical anesthesia, lidocaine is used following as for standard FBO $(1,64)$. In nonintubated patients, the patient's agitation may lead to desaturation and compromise the realization of FBO during ppNIMV. Chiner et al. (65) demonstrated the feasibility and safety of a target-controlled (TCI) propofol sedation during $B A L$ in a series of 23 patients with hypoxemic ARF (mean $\mathrm{PaO} 2 / \mathrm{FiO} 2$ pre-FBO $=181$ $\mathrm{mmHg}$ ). All patients tolerated $\mathrm{FBO}$ with $\mathrm{BAL}$ and none was intubated during the 2 hour after the procedure. Loss of consciousness was obtained with an effect site concentration of propofol of $1.49 \pm 0.46 \mu \mathrm{g} / \mathrm{mL}$. TCl propofol allowed to obtain amnesia, patient comfort, and it did not impair airway protection. Any hemodynamic changes observed were modest and transient. Even if analgesics (opioids) and/or sedatives (benzodiazepines, propofol) could be helpful for FBO under ppNIMV to reduce patient's discomfort, it's important to highlight that clinicians must have experience in drug management and airway management (66).

It is recommended to carry out the procedure with the patient in a semi-recumbent position, which is implied by the anterior entry of the bronchoscope. However, ppNIMV itself has been used in supine decubitus in order to optimize respiratory parameters in other explorations like interventional cardiology or in suspected dynamic airway collapse $(16,25,34)$. As in all interventions in high-risk patients, it is advisable to take 


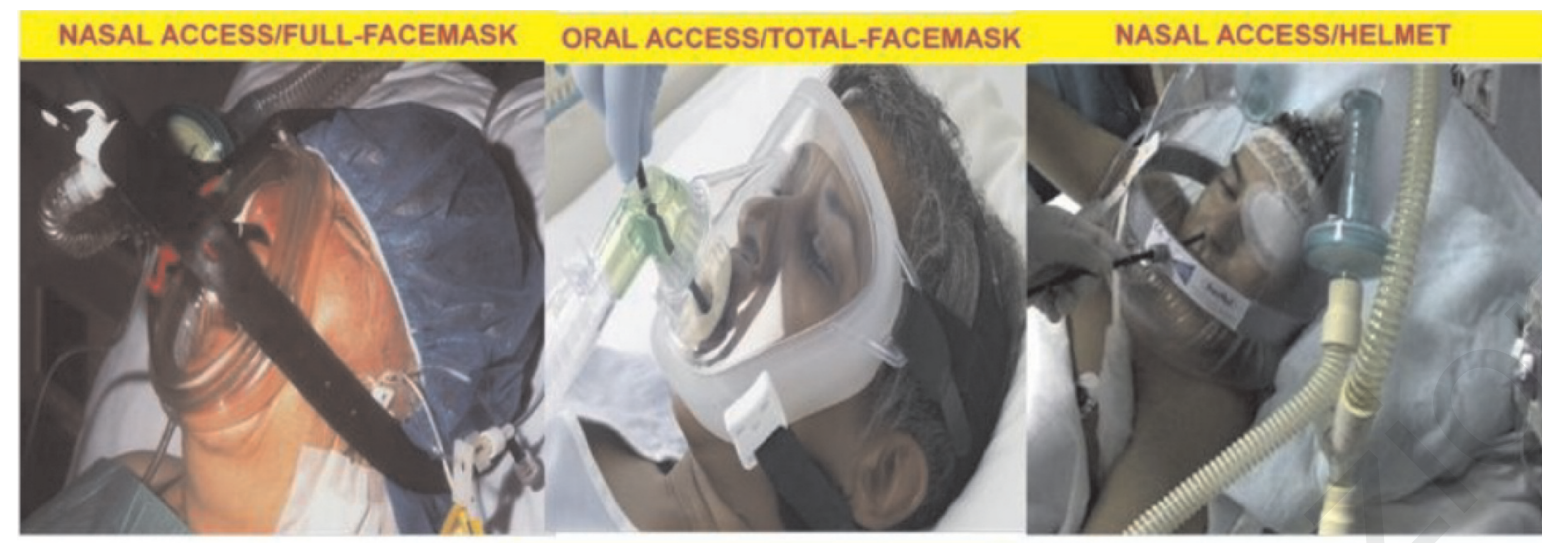

ORAL ACCEES /FULL-FACEMASK

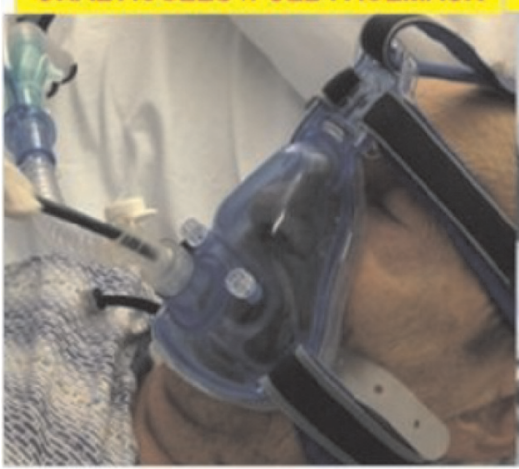

ORAL ACCESS/NASAL MASK

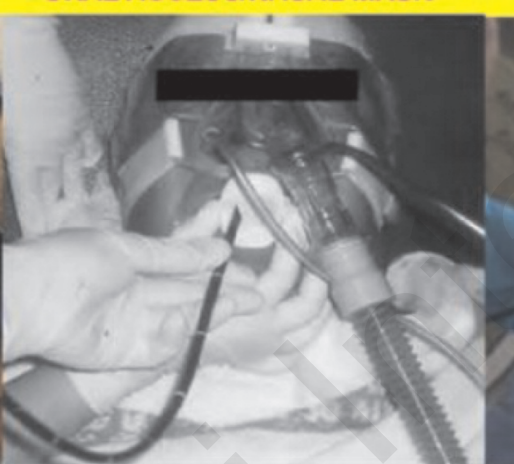

ORAL ACCESS/TOTAL-FACE MASK

Figure 3 - Different combinations of access of bronchoscope into the airways (nasal or oral route) and interfaces (nasal, fullface, total-face mask and helmet) used for delivering ppNIMV.

\begin{abstract}
Risks of the combined NIMV-bronchoscopy procedure should be not underestimated and, in this context, the role of the nursing team in preventing complications is fundamental.
\end{abstract}

the shortest time possible under 10 min (25). ppNIMV will be maintained with a setting similar to those prior to FBO within 15 and 90 min after the procedure (25).

Risks of the combined NIMV-bronchoscopy procedure should be not underestimated and, in this context, the role of nursing team in preventing complications is fundamental (67). ppNIMV may induce gastro-distension and increased risk for pulmonary aspiration; the abdominal pressure can reduce functional residual capacity and increase the elastic work of breathing $(9,16,34)$. Lower pressures of the ventilator and semi-recumbent patient's position may reduce this risk (25). Nasal or facial injuries appear only after a prolonged NIMV use (13). There are also other complications related with FBO (gas exchange derangement, bleeding, poor collaboration, etc.), whose resolution is approached as in a standard FBO with prompt ETI availability $(1,2)$. The rate of pneumothorax after TBLB is considerably higher during CMV than in spontaneously breathing patients $(14,3-23,6$ vs $<5 \%)(68,69)$. Conversely, we have no data about the rate of pneumothorax during NIMV-assisted TBLB. However, the risk should not to be under- estimated as pneumothorax was reported during both in acute and chronic ppNIMV $(70,71)$. Accordingly, facilities for inserting a chest drainage should be promptly available. Less frequently, there may be major cardiovascular complications (e.g. malignant arrhythmias, acute coronary syndrome, cardiac arrest), which can be minimized through proper patient selection and monitoring during bronchoscopy $(1,2,25)$. Contraindications for FBO-NIMV combined procedures are those of ppNIMV itself, such as cardiac arrest, severe encephalopathy, gastrointestinal bleeding, severe hemodynamic instability, history of facial surgery or trauma, recent esophageal-gastric interventions. inability to protect the airway $(9,16)$. In addition to that, there are other absolute contraindications of bronchoscopy itself, such as, recent acute coronary syndrome, severe arrhythmias, and severe coagulopathies if biopsy is planned $(1,2)$.

\section{Conclusions}

An increasing amount of data suggested the use of bronchoscopy during NIMV in ARF in order to avoid/reduce the need of ETI. Despite a strong rationale for the combined use of the two techniques, there is not still enough evidence for a large-scale application of this strategy in all clinical scenarios. The majority of the available data are in favor of the "help" given 


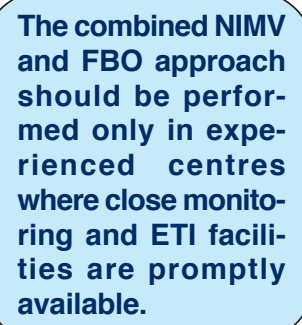

Other interesting potential ETI, OSAS and pediatric patients, as well as candidates for interventional RB procedures. This combined approach should be performed only in centres showing wide experience with both NIMV and bronchoscopy, where close monitoring and ETI facilities are promptly available.

\section{References}

1. Du Rand IA, Blaikley J, Booton R, Chaudhuri N, Gupta V, Khalid S, et al.; British Thoracic Society Bronchoscopy Guideline Group. British Thoracic Society guideline for diagnostic flexible bronchoscopy in adults: accredited by NICE. Thorax. 2013;68 Suppl 1:i1-i44.

2. Dellinger RP, Bandi V. Fiberoptic bronchoscopy in the intensive care unit. Crit Care Clin. 1992;8:755-72.

3. Wain JC. Rigid bronchoscopy: the value of a venerable procedure. Chest Surg Clin N Am. 2001; 11:6919.

4. Wahidi MM, Herth FJ, Ernst A. State of the art: interventional pulmonology. Chest. 2007;131:261-74. 5.

5. Ikeda S, Yanai N, Ishikawa S. Flexible bronchoscope. Keio J Med. 1968;17:1.

6. Lim WS, Baudouin SV, George RC, Hill AT, Jamieson C, Le Jeune I, et al. Pneumonia Guidelines Committee of the BTS Standards of Care Committee. British Thoracic Society guidelines for the management of community acquired pneumonia in adults: update 2009. Thorax. 2009;64(Suppl III):iii1-iii55.

7. Ramsdell J, Narsavage GL, Fink JB; American College of Chest Physicians' Home Care Network Working Group. Management of community-acquired pneumonia in the home: an American College of Chest Physicians clinical position statement. Chest. 2005 May;127(5):1752-63.

8. Bradley B, Branley HM, Egan JJ, Greaves MS, Hansell DM, Harrison NK, et al; British Thoracic Society Interstitial Lung Disease Guideline Group, British Thoracic Society Standards of Care Committee; Thoracic Society of Australia; New Zealand Thoracic Society; Irish Thoracic Society. Interstitial lung disease guideline: the British Thoracic Society in collaboration with the Thoracic Society of Australia and New Zealand and the Irish Thoracic Society. Thorax. 2008 Sep;63 Suppl 5:v1-58.

9. Nava S, Hill N. Non-invasive ventilation in acute respiratory failure. Lancet. 2009;374:250-9.
10. Corrado A, Gorini M. Negative-pressure ventilation: is there still a role? Eur Respir J. 2002;20:187-97.

11. International Consensus Conference in Intensive Care Medicine: non-invasive positive pressure ventilation in acute respiratory failure. Am J Respir Crit Care Med. 2001;163:283-91.

12. Scala R, Naldi M. Ventilators for noninvasive ventilation to treat acute respiratory failure. Respir Care. 2008;53:1054-80.

13. Nava S, Navalesi P, Gregoretti C. Interfaces and humidification for noninvasive mechanical ventilation. Respir Care. 2009;54:71-84.

14. Lindholm CE, Ollman B, Snyder J, Millen E, Grenvik A. Flexible fiberoptic bronchoscopy in critical care medicine. Diagnosis, therapy and complications. Crit Care Med. 1974;2:250-61.

15. Credle Jr WF, Smiddy JF, Shea DW, Elliott RC. Fiberoptic bronchoscopy in acute respiratory failure in the adult. N Engl J Med. 1973;288:49-50.

16. Murgu SD, Pecson J, Colt HG. Bronchoscopy during noninvasive ventilation: indications and technique. Respir Care. 2010;55:595-600.

17. Dreher M, Ekkernkamp E, Storre JH, Kabitz HJ, Windisch W. Sedation during flexible bronchoscopy in patients with pre-existing respiratory failure: Midazolam versus Midazolam plus Alfentanil. Respiration. 2010;79:307-14.

18. Golpe R, Mateos A. Supplemental oxygen during flexible bronchoscopy. Chest. 2002; 121:663-4.

19. Payne CB Jr, Goyal PC, Gupta SC. Effects of transoral and transnasal fiberoptic bronchoscopy on oxygenation and cardiac rhythm. Endoscopy. 1986;18:13.

20. Katz AS, Michelson EL, Stawicki J, Holford FD. Cardiac arrhythmias, frequency during fiberoptic bronchoscopy and correlation with hypoxemia. Arch Intern Med. 1981;141:603-606.

21. Azoulay E, Mokart D, Lambert J, Lemiale V, Rabbat A, Kouatchet A, et al. Diagnostic strategy for hematology and oncology patients with acute respiratory failure: randomized controlled trial. Am J Respir Crit Care Med. 2010;182:1038-1046.

22. Cracco C, Fartoukh M, Prodanovic H, Azoulay E, Chenivesse C, Lorut C, et al. Safety of performing fiberoptic bronchoscopy in critically ill hypoxemic patients with acute respiratory failure. Intensive Care Med. 2013;39:45-52.

23. Goldstein RA, Rohatgi PK, Bergofsky EH, Block ER, Daniele RP, Dantzker DR, et al, American Thoracic Society. Clinical role of bronchoalveolar lavage in adults with pulmonary disease. Am Rev Respir Dis. 1990;142:481-6.

24. Ambrosino N, Vagheggini G. Noninvasive positive pressure ventilation in the acute care setting: where are we? Eur Respir J. 2008;31:874-86.

25. Esquinas A, Zuil M, Scala R, Chiner E. Bronchoscopy during non-invasive mechanical ventilation: a review of techniques and procedures. Arch Bronconeumol. 2013;49:105-12.

26. Cavaliere S, Venuta F, Foccoli P, Toninelli C, La Face B. Endoscopic treatment of malignant airway 
obstructions in 2008 patients. Chest. 1996;11:153642.

27. Vitacca M, Natalini G, Cavaliere S, Clini E, Foccoli P, Candiani A, et al. Breathing pattern and arterial blood gases during Nd-YAG laser photoresection of endobronchial lesions under general anesthesia: use of negative pressure ventilation: a preliminary study. Chest. 1997;112:1466-73.

28. Ozyilmaz E, Ugurlu AO, Nava S. Timing of noninvasive ventilation failure: causes, risk factors, and potential remedies. BMC Pulm Med. 2014;14:19.

29. Demoule A, Girou E, Richard JC, Taille S, Brochard L. Benefits and risks of success or failure of noninvasive ventilation. Intensive Care Med. 2006;32: 1756-65.

30. Carlucci A, Richard JC, Wysocki M, Lepage E, Brochard L. SRLF Collaborative Group on Mechanical Ventilation. Noninvasive versus conventional mechanical ventilation. An epidemiologic survey. Am J Respir Crit Care Med. 2001;163: 874- 880.

31. Scala R, Naldi M, Archinucci I, Coniglio G, Nava S. Noninvasive positive pressure ventilation in patients with acute exacerbations of COPD and varying levels of consciousness. Chest. 2005;128: 1657-66.

32. Scala R, Nava S, Conti G, Antonelli M, Naldi M, Archinucci I, et al. Non-invasive versus conventional ventilation to treat hypercapnic encephalopathy in COPD. Intensive Care Med. 2007;33:2101-8.

33. Conti G, Antonelli M, Navalesi P, Rocco M, Bufi M, Spadetta G, et al. Noninvasive vs. conventional mechanical ventilation in patients with chronic obstructive pulmonary disease after failure of medical treatment in the ward: a randomized trial. Intensive Care Med. 2002;28:1701-7.

34. Ambrosino N, Guarracino F. Unusual applications of non-invasive ventilation. Eur Respir J. 2011;38: 440-9.

35. Scala R, Nava S. NIV and palliative care. Eur Respir Mon. 2008;41:287-306.

36. Antonelli M, Conti G, Riccioni L, Meduri GU. Noninvasive positive-pressure ventilation via face mask during bronchoscopy with BAL in high-risk hypoxemic patients. Chest. 1996;110:724-8.

37. Da Conceiçao M, Genco G, Favier JC, Bidallier I, Pitti R. Fiberoptic bronchoscopy during noninvasive positive-pressure ventilation in patients with chronic obstructive lung disease with hypoxemia and hipercapnia. Ann Fr Anesth Reanim. 2000;19:231-6.

38. Maitre B, Jaber S, Maggiore SM, Bergot E, Richard $\mathrm{JC}$, Bakthiari $\mathrm{H}$, et al. Continuous positive airway pressure during fiberoptic bronchoscopy in hypoxemic patients. A randomized double-blind study using a new device. Am J Respir Crit Care Med. 2000;162:1063-7.

39. Antonelli M, Conti G, Rocco M, Arcangeli A, Cavaliere F, Proietti R, et al. Noninvasive positive-pressure ventilation vs conventional oxygen supplementation in hypoxemic patients undergoing diagnostic bronchoscopy. Chest. 2002;121:1149-54.

40. Antonelli M, Pennisi MA, Conti G, Bello G, Maggiore SM, Michetti V, Cavaliere F, et al. Fiberoptic bronchoscopy during noninvasive positive pressure ven- tilation delivered by helmet. Intensive Care Med. 2003;29:126-9.

41. Chiner E, Sancho-Chust JN, Llombart M, Senent C, Camarasa A, Signes-Costa J. Fiberoptic bronchoscopy during nasal non-invasive ventilation in acute respiratory failure. Respiration. 2010;80:3216.

42. Heunks LM, de Bruin CJ, van der Hoeven JG, van der Heijden HF. Noninvasive mechanical ventilation for diagnostic bronchoscopy using a new face mask: an observational feasibility study. Intensive Care Med. 2010;36:143-7.

43. Baumann HJ, Klose H, Simon M, Ghadban T, Braune SA, Hennigs JK, et al. Fiberoptic bronchoscopy in patients with acute hypoxemic respiratory failure requiring noninvasive ventilation-a feasibility study. Crit Care. 2011;15:R179.

44. Agarwal R, Khan A, Aggarwal AN, Gupta D. Bronchoscopic lung biopsy using noninvasive ventilatory support: case series and review of literature of NIV-assisted bronchoscopy. Respir Care. 2012;57: 1927-36.

45. Jaber S, Chanques C. Another step for noninvasive ventilation in chronic obstructive pulmonary disease patients. Crit Care. 2010;14:163-4.

46. Scala R, Naldi M, Maccari U. Early fiberoptic bronchoscopy during noninvasive ventilation in patients with decompensated chronic obstructive pulmonary disease due to community acquired pneumonia. Crit Care. 2010;14:R80.

47. Maggiorelli C, Ciarleglio G, Granese V, Maccari U, Manta C, Madioni $\mathrm{C}$, et al. Integrated therapeutic strategy during noninvasive ventilation in a patient with end-stage respiratory disease. Respir Care. $2015 \mathrm{Apr} ; 60(4)$ :e80-5.

48. Weiss YG, Deutschman CS. The role of fiberoptic bronchoscopy in airway management of the critically ill patient. Crit Care Clin. 2000;16:445-51.

49. Bourgain JL, Billard V, Cros AM. Pressure support ventilation during fibreoptic intubation under propofol anaesthesia. Br J Anaesth. 2007;98:136-40.

50. Jaber S, Amraoui J, Lefrant JY, Arich C, Cohendy R, Landreau $\mathrm{L}$, et al. Clinical practice and risk factors for immediate complications of endotracheal intubation in the intensive care unit: a prospective, multiple-center study. Crit Care Med. 2006; 34:2355-61.

51. Delclaux C, L'Her E, Alberti C, Mancebo J, Abroug F, Conti G, et al. Treatment of acute hypoxemic nonhypercapnic respiratory insufficiency with continuous positive airway pressure delivered by a face mask: A randomized controlled trial. JAMA. 2000;284:2352-60.

52. Baillard C, Fosse JP, Sebbane M, Chanques G, Vincent F, Courouble P, et al. Noninvasive ventilation improves preoxygenation before intubation of hypoxic patients. Am J Respir Crit Care Med. 2006; 174:171-7.

53. Da Conceiçao M, Favier JC, Bidallier I, Armanet L, Steiner T, Genco G, et al. Fiber-optic intubation with non-invasive ventilation with an endoscopic facial mask. Ann Fr Anesth Reanim. 2002;21:256-62.

54. Barjaktarevic I, Berlin D. Bronchoscopic intubation during continuous nasal positive pressure ventilation in the treatment of hypoxemic respiratory failure. $\mathrm{J} \mathrm{In-}$ 
tensive Care Med. 2015;30:161-6.

55. Corso RM, Gregoretti C, Braghiroli A, Fanfulla F, Insalaco G. Practice Guidelines for the Perioperative Management of Patients with Obstructive Sleep Apnea: navigating through uncertainty. Anesthesiology. 2014;121:664-5.

56. Borowiecki B, Pollak CP, Weitzman ED, Rakoff S, Imperato J. Fibro-optic study of pharyngeal airway during sleep in patients with hypersomnia obstructive sleep-apnea syndrome. Laryngoscope. 1978;88: 1310-3.

57. Rothfleisch R, Davis LL, Kuebel DA, deBoisblanc BP. Facilitation of fiberoptic nasotracheal intubation in a morbidly obese patient by simultaneous use of nasal CPAP. Chest. 1994;106:287-8.

58. Schnapf BM. Oxygen desaturation during fiberoptic bronchoscopy in pediatric patients. Chest. 1991; 99:591-4.

59. Kuna ST, Bedi DG, Ryckman C. Effect of nasal airway positive pressure on upper airway size and configuration. Am Rev Respir Dis. 1988;138:969-75.

60. Trachsel D, Erb TO, Frei FJ, Hammer J, Swiss Paediatric Respiratory Research Group. Use of continuous positive airway pressure during flexible bronchoscopy in young children. Eur Respir J. 2005; 26:773-7.

61. Panitch HB, Allen JL, Alpert BE, Schidlow DV. Effects of CPAP on lung mechanics in infants with acquired tacheobronchomalacia. Am J Respir Crit Care Med. 1994;150:1341-6.

62. Natalini G, Cavaliere S, Vitacca M, Amicucci G, Ambrosino N, Candiani A. Negative pressure ventilation vs. spontaneous assisted ventilation during rigid bronchoscopy. A controlled randomised trial. Acta Anaesthesiol Scand. 1998;42:1063-9.
63. Natalini G, Cavaliere S, Seramondi V, Foccoli P, Vitacca M, Ambrosino N. et al. Negative pressure ventilation vs external high frequency oscillation during rigid bronchoscopy. A controlled randomized trial. Chest. 2000;118:18-23.

64. Reed A. Preparation of the patient for awake flexible fiberoptic bronchoscopy. Chest. 1992;101:813-6.

65. Chiner E, Bui HN, Guilhon E, Grenouillet-Delacre M, Leger MS, Saghi T, et al. Fiberoptic bronchoscopy under noninvasive ventilation and propofol targetcontrolled infusion in hypoxemic patients. Intensive Care Med. 2011;37:1969-75.

66. Scala R. Sedation during non-invasive ventilation to treat acute respiratory failure. Shortness of Breath. 2013;2:35-43.

67. Murgu SD, Pecson J, Colt HG. Flexible bronchoscopy assisted by noninvasive positive pressure ventilation. Crit Care Nurse. 2011;31:70-6.

68. Carron M, Gagliardi G, Michielan F, Freo U, Ori C. Occurrence of pneumothorax during noninvasive positive pressure ventilation through a helmet. J Clin Anesth. 2007;19:632-5.

69. Choo-Kang LR, Ogunlesi FO, McGrath-Morrow SA, Crawford TO, Marcus CL. Recurrent pneumothoraces associated with nocturnal noninvasive ventilation in a patient with muscular dystrophy. Pediatr Pulmonol. 2002;34:73-8.

70. Bulpa PA, Dive AM, Mertens L, Delos MA, Jamart $J$, Evrard PA, et al. Combined bronchoalveolar lavage and transbronchial lung biopsy: safety and yield in ventilated patients. Eur Respir J. 2003; 21: 489-94.

71. O'Brien JD, Ettinger NA, Shevlin D, Kollef MH. Safety and yield of transbronchial biopsy in mechanically ventilated patients. Crit Care Med. 1997;25:440-6. 